Article

\title{
On the High-Power Mean of the Generalized Gauss Sums and Kloosterman Sums
}

\section{Xinyu Liu and Wenpeng Zhang *}

School of Mathematics, Northwest University, Xi'an 710127, China; liuxinyu@stumail.nwu.edu.cn

* Correspondence: wpzhang@nwu.edu.cn

Received:15 August 2019; Accepted: 25 September 2019; Published: 27 September 2019

check for updates

\begin{abstract}
The main aim of this paper is to use the properties of the trigonometric sums and character sums, and the number of the solutions of several symmetry congruence equations to research the computational problem of a certain sixth power mean of the generalized Gauss sums and generalized Kloosterman sums, and to give two exact computational formulae for them.
\end{abstract}

Keywords: symmetry congruence equation; generalized Gauss sums; generalized Kloosterman sums; sixth power mean; computational formula

MSC: 11L03; 11L07

\section{Introduction}

Let $q \geq 3$ be a positive integer, $m$ and $n$ be integers. Then for any positive integers $r>s \geq 1$ and Dirichlet character $\chi \bmod q$, the generalized Gauss sums $G(m, n, r, s, \chi ; q)$ is defined as

$$
G(m, n, r, s, \chi ; q)=\sum_{a=1}^{q} \chi(a) e\left(\frac{m a^{r}+n a^{s}}{q}\right)
$$

where $e(y)=e^{2 \pi i y}$.

It is well known that this sum plays an extremely essential role in the research of analytic number theory, and plenty of classical problems in analytic number theory are closely related to it. For instance, if $q=p$ is an odd prime, $r=p-2$ and $s=1$, then

$$
G(m, n, p-2,1, \chi ; p)=\sum_{a=1}^{p-1} \chi(a) e\left(\frac{m \bar{a}+n a}{q}\right)
$$

becomes the well-known Kloosterman sum, where $a \cdot \bar{a} \equiv 1 \bmod p$, see H. Iwaniec's book [1] or Zhang Wenpeng's papers [2-5]. Therefore, any substantial advance in the study of $G(m, n, r, s, \chi ; q)$ will certainly promote the development of multiplicative number theory and analytic number theory. Due to these reasons, a number of scholars have research the properties of $G(m, n, r, s, \chi ; q)$, and obtained various vital results. For instance, Zhang Han and Zhang Wenpeng [6] proved that for any odd prime $p$, one has the identity

$$
\sum_{m=1}^{p-1}\left|\sum_{a=0}^{p-1} e\left(\frac{m a^{3}+n a}{p}\right)\right|^{4}= \begin{cases}2 p^{3}-p^{2} & \text { if } 3 \nmid p-1, \\ 2 p^{3}-7 p^{2} & \text { if } 3 \mid p-1,\end{cases}
$$

where $n$ represents any integer with $(n, p)=1$. 
Zhang Wenpeng and Han Di [7] acquired the identity

$$
\sum_{a=1}^{p-1}\left|\sum_{n=0}^{p-1} e\left(\frac{n^{3}+a n}{p}\right)\right|^{6}=5 p^{4}-8 p^{3}-p^{2},
$$

where $p$ denotes an odd prime with $3 \nmid(p-1)$.

Duan Ran and Zhang Wenpeng [8] proved that for any prime $p$ with $3 \nmid(p-1)$, and any Dirichlet character $\lambda \bmod p$, one has the identity

$$
\sum_{m=1}^{p-1}\left|\sum_{a=1}^{p-1} \lambda(a) e\left(\frac{m a^{3}+n a}{p}\right)\right|^{4}= \begin{cases}3 p^{3}-8 p^{2} & \text { if } \lambda=\left(\frac{*}{p}\right) \\ 2 p^{3}-7 p^{2} & \text { if } \lambda \neq \chi_{0} \\ 2 p^{3}-3 p^{2}-3 p-1 & \text { if } \lambda=\chi_{0}\end{cases}
$$

where $\left(\frac{*}{p}\right)$ denotes the Legendre symbol and $\chi_{0}$ is the principal character mod $p$.

Several other outcomes related to exponential sums and Kloostermann sums can also be found in the references [9-16]. These contents will not be repeated here.

In this paper, we mainly take into account the computational problems of the $2 k$-th power mean of the generalized Gauss sum and generalized Kloosterman sum. In other words,

$$
\sum_{\chi \bmod } \sum_{p}^{p-1}\left|\sum_{a=1}^{p-1} \chi(a) e\left(\frac{m a^{3}+a}{p}\right)\right|^{2 k}
$$

and

$$
\sum_{\chi \bmod } \sum_{p=0}^{p-1}\left|\sum_{a=1}^{p-1} \chi(a) e\left(\frac{m a+\bar{a}}{p}\right)\right|^{2 k}
$$

where $k \geq 3$ represents an integer.

Regarding $2 k$-th power means (1) and (2) with $k \geq 3$, up to now no one seems to research them, and we have not seen any relevant papers before. These problems make a lot of sense. In addition to reflecting the value distribution properties of generalized Gauss sums and Kloosterman sums themselves, they also have many important applications in analytic number theory. For example, Zhang Yitang's very important works [17] on the gaps between primes is obtained based on the sieve methods and some special mean value estimate for Kloosterman sums. Therefore, this research is necessary, which is also the original intention of our paper.

The main objective of this paper is to apply the properties of the trigonometric sums and character sums, and the number of the solutions of several congruence equations to research the computational problem of (1) and (2) for $k=3$, and give two exact computational formulae for them. In other words, we are going to prove the following:

Theorem 1. For any odd prime $p$ with $3 \nmid(p-1)$, we have the identity

$$
\begin{aligned}
\sum_{\chi \bmod } \sum_{p=0}^{p-1}\left|\sum_{a=1}^{p-1} \chi(a) e\left(\frac{m a^{3}+a}{p}\right)\right|^{6} & =\sum_{\chi \bmod } \sum_{m=0}^{p-1}\left|\sum_{a=1}^{p-1} \chi(a) e\left(\frac{a^{3}+m a}{p}\right)\right|^{6} \\
& =p(p-1)\left(6 p^{3}-28 p^{2}+39 p+5\right) .
\end{aligned}
$$


Theorem 2. For any odd prime $p$, we have the identity

$$
\sum_{\chi \bmod } \sum_{p}^{p-1}\left|\sum_{a=1}^{p-1} \chi(a) e\left(\frac{m a+\bar{a}}{p}\right)\right|^{6}=p(p-1)\left(5 p^{3}-18 p^{2}+14 p+5\right) .
$$

According to these results, we may instantly deduce the following two corollaries:

Corollary 1. For any odd prime $p$ with $3 \nmid(p-1)$, there exists an integer $1 \leq m \leq p-1$ and a non-principal character $\chi$ mod $p$ such that the following inequality holds,

$$
\left|\sum_{a=1}^{p-1} \chi(a) e\left(\frac{m a^{3}+a}{p}\right)\right|>6^{\frac{1}{6}} \cdot p^{\frac{1}{2}}-\frac{1}{\sqrt{p}} .
$$

Corollary 2. For any odd prime $p$, there exists an integer $1 \leq m \leq p-1$ and a non-principal character $\chi \bmod p$ and we can get the inequality

$$
\left|\sum_{a=1}^{p-1} \chi(a) e\left(\frac{m a+\bar{a}}{p}\right)\right|>5^{\frac{1}{6}} \cdot p^{\frac{1}{2}}-\frac{1}{\sqrt{p}} .
$$

Open problems: in Theorem 1, we merely discussed the special case $3 \nmid(p-1)$. Suppose that $3 \mid(p-1)$, the situation will be more complicated. We have not found an effective approach to calculate it up to now, so it is also an open problem.

For $3 \nmid(p-1)$ and $k=4$, applying our method, we do not seem to be able to acquire an exact computational formula for (1) or (2). These are also two thoughtful problems. Interested readers can give them a try.

\section{Some Lemmas}

In this part, we are going to introduce four uncomplicated lemmas. These are actually gained by a certain decomposition in the process of theorem proving. Of course, plenty of elementary number theory knowledge required for the following arguments can be found in reference [18], we will not repeat them here. At first, we have the following:

Lemma 1. Let $p$ be an odd prime with $3 \nmid(p-1)$, then we have the identity

$$
\begin{aligned}
& \sum_{a=1}^{p-1} \sum_{b=1}^{p-1} \sum_{c=1}^{p-1} \sum_{d=1}^{p-1} \sum_{e=1}^{p-1} 1=p^{3}-3 p^{2}+5 p-5 . \\
& a^{3}+b^{3}+c^{3} \equiv d^{3}+e^{3}+1(\bmod p) \\
& a b c \equiv \operatorname{de}(\bmod p)
\end{aligned}
$$

Proof. According to the properties of the reduced residue system $\bmod p$, we are aware of that if $d$ and $e$ pass through a reduced residue system mod $p$ respectively, then $d a$ and $e b$ also pass through a reduced residue system $\bmod p$ for all $1 \leq a \leq p-1$ and $1 \leq b \leq p-1$. Since $3 \nmid(p-1)$, if $a$ passes through a reduced residue system $\bmod p$, then $a^{3}$ also passes through a reduced residue system mod $p$. Combining these properties, we obtain

$$
\begin{aligned}
& \sum_{\substack{a=1 \\
a^{3}+b^{3}+c^{3} \equiv d^{3}+e^{3}+1(\bmod p)}}^{p-1} \sum_{c=1}^{p-1} \sum_{d=1}^{p-1} \sum_{e=1}^{p-1} 1=\sum_{\substack{a=1 \\
a^{3}+b^{3}+c^{3} \equiv a^{3} d^{3}+b^{3} e^{3}+1(\bmod p)}}^{p-1} \sum_{c=1}^{p-1} \sum_{d=1}^{p-1} \sum_{e=1}^{p-1} 1 \\
& a b c \equiv d e(\bmod p) \quad c \equiv d e(\bmod p)
\end{aligned}
$$




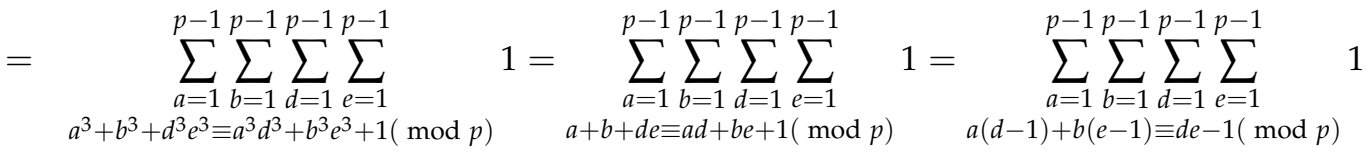

$$
\begin{aligned}
& =\sum_{a=1}^{p-1} \sum_{b=1}^{p-1} 1+2 \sum_{\substack{a=1 \\
b(e-1) \equiv e-1(\bmod p)}}^{p-1} \sum_{b=1}^{p-1} \sum_{e=2}^{p-1}+\sum_{\substack{a=1 \\
a(d-1)+b(e-1) \equiv d e-1(\bmod p)}}^{p-1} \sum_{b=1}^{p-1} \sum_{d=2}^{p-1} \sum_{e=2}^{p-1} 1 \\
& =(p-1)^{2}+2(p-1)(p-2)+\sum_{\substack{a=0 \\
a+b \equiv d e-1(\bmod p)}}^{p-1} \sum_{\substack{p-1 \\
d=2}}^{p-1} \sum_{e=2}^{p-1} 1-\sum_{\substack{b=1 \\
b \equiv d e-1(\bmod p)}}^{p-1} \sum_{d=2}^{p-1} \sum_{e=2}^{p-1} 1 \\
& =(p-1)(3 p-5)+(p-1)(p-2)^{2}-\left((p-2)^{2}-(p-2)\right) \\
& =p^{3}-3 p^{2}+5 p-5 \text {. }
\end{aligned}
$$

This proves Lemma 1.

Lemma 2. Let $p$ be an odd prime, then we have the identity

$$
\sum_{\substack{a=1 \\ a+b+c \equiv d+e+1(\bmod p) \\ a b c \equiv d e(\bmod p)}}^{p-1} \sum_{\substack{c=1 \\ a b c}}^{p-1} \sum_{d=1}^{p-1} 1=p^{3}-3 p^{2}+5 p-5 .
$$

Proof. According to the method of proving Lemma 1, we can easily deduce that

$$
\sum_{\substack{a=1 \\ a+b+c \equiv d+e+1(\bmod p) \\ a b c \equiv d e(\bmod p)}}^{p-1} \sum_{\substack{c=1 \\ a b c}}^{p-1} \sum_{\substack{d=1 \\ p-1}}^{p-1} 1=\sum_{\substack{a=1 \\ a+b+d e \equiv a d+b e+1(\bmod p)}}^{p-1} \sum_{b=1}^{p-1} \sum_{d=1}^{p-1} \sum_{e=1}^{p-1} 1=p^{3}-3 p^{2}+5 p-5 .
$$

This proves Lemma 2.

Lemma 3. Let $p$ is an odd prime with $3 \nmid(p-1)$, then we have the identity

$$
\begin{gathered}
\sum_{a=1}^{p-1} \sum_{b=1}^{p-1} \sum_{c=1}^{p-1} \sum_{d=1}^{p-1} \sum_{e=1}^{p-1} 1=7 p^{2}-31 p+44 . \\
a^{3}+b^{3}+c^{3} \equiv d^{3}+e^{3}+1(\bmod p) \\
a b c \equiv d e(\bmod p) \\
a+b+c \equiv d+e+1(\bmod p)
\end{gathered}
$$

Proof. First note that the conditions $a^{3}+b^{3}+c^{3} \equiv d^{3}+e^{3}+1(\bmod p), a+b+c \equiv d+e+1(\bmod p)$ and $a b c \equiv d e(\bmod p)$ are equivalent to $(a+b+c)^{3}-a^{3}-b^{3}-c^{3} \equiv(d+e+1)^{3}-\left(d^{3}+e^{3}+1\right)(\bmod$ $p), a+b+c \equiv d+e+1(\bmod p)$ and $a b c \equiv d e(\bmod p)$. These conditions are equivalent to $a^{2}(b+$ $c)+b^{2}(a+c)+c^{2}(a+b) \equiv d^{2}(e+1)+e^{2}(d+1)+(d+e)(\bmod p), a+b+c \equiv d+e+1(\bmod p)$ and $a b c \equiv d e(\bmod p)$, they are equivalent to $a^{2}(d+e+1-a)+b^{2}(d+e+1-b)+c^{2}(d+e+1-$ $c) \equiv d^{2}(a+b+c-d)+e^{2}(a+b+c-e)+(a+b+c-1)(\bmod p), a+b+c \equiv d+e+1(\bmod p)$ and $a b c \equiv d e(\bmod p)$, or they are equivalent to $(a+b+c)\left(a^{2}+b^{2}+c^{2}-d^{2}-e^{2}-1\right) \equiv 0(\bmod p)$, $a+b+c \equiv d+e+1(\bmod p)$ and $a b c \equiv d e(\bmod p)$.

It is clear that $(a+b+c)\left(a^{2}+b^{2}+c^{2}-d^{2}-e^{2}-1\right) \equiv 0(\bmod p)$ is equivalent to $a+b+c \equiv$ $0(\bmod p)$ or $a^{2}+b^{2}+c^{2}-d^{2}-e^{2}-1 \equiv 0(\bmod p)$. 
First case: if $a+b+c \equiv 0(\bmod p)$, then from the properties of the reduced residue system $\bmod p$ and note that if $(3, p-1)=1$ and $c$ pass through a reduced residue system $\bmod p$, then $c^{3}$ also pass through a reduced residue system $\bmod p$, so we have

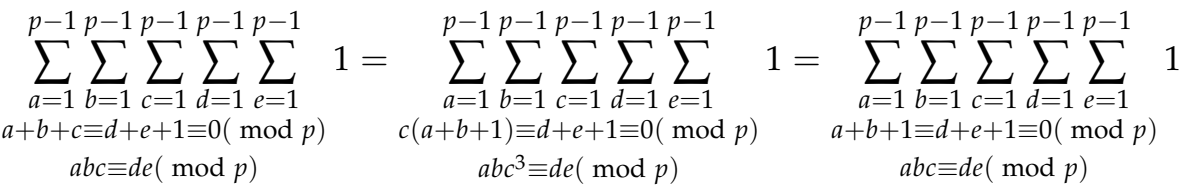

$$
\begin{aligned}
& =\sum_{\substack{a=1 \\
a+b+1 \equiv d+e+1 \equiv 0(\bmod p)}}^{p-1} \sum_{\substack{p-1 \\
b=1}}^{p-1} \sum_{\substack{p-1 \\
e=1 \\
a+b+1 \equiv 0(\bmod p)}}^{p-1} \sum_{\substack{p-1 \\
b=1}}^{p-1}=(p-2)^{2} .
\end{aligned}
$$

Second case: if $a^{2}+b^{2}+c^{2}-d^{2}-e^{2}-1 \equiv 0(\bmod p)$, then we have

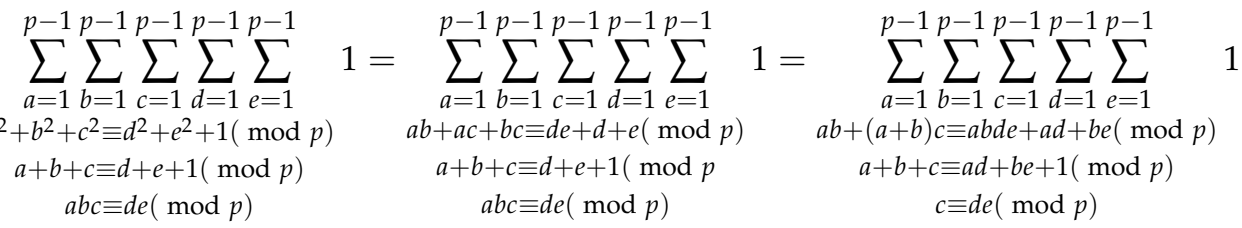

$$
\begin{aligned}
& =\sum_{\substack{a=1 \\
(a-1)(b-1)(d e-1) \equiv 0(\bmod p)}}^{p-1} \sum_{b=1}^{p-1} \sum_{d=1}^{p-1} p-1=1+\sum_{\substack{d=1 \\
(d-1)(e-1) \equiv 0(\bmod p)}}^{p-1} \sum_{\substack{e=1 \\
(d-1)(b-\bar{d}) \equiv 0(\bmod p)}}^{p-1} 1+2 \sum_{\substack{b=2 \\
d=1}}^{p-1} 1 \\
& \begin{array}{ccc}
(a-1)(b-1)(d e-1) \equiv 0(\bmod p) & (d-1)(b-\bar{d}) \equiv 0(\bmod p) \\
a+b+d e \equiv a d+b e+1(\bmod p) & (d e-1, p)=1
\end{array} \\
& +2 \sum_{\substack{b=2 \\
(e-1)(b-d) \equiv 0(\bmod p) \\
(d e-1, p)=1}}^{p-1} \sum_{\substack{d=1 \\
(d e=1}}^{p-1} 1+\sum_{\begin{array}{c}
a=2 \\
a+b \equiv a d+b e(\bmod p) \\
d e \equiv 1(\bmod p)
\end{array}}^{p-1} \sum_{b=2}^{p-1} \sum_{d=1}^{p-1} \sum_{e=1}^{p-1} 1 \\
& =1+2(p-2)+4(p-2)+2(p-2)(2 p-5)+\sum_{\substack{a=2 \\
(a+b) e \equiv a+b e^{2}(\bmod p)}}^{p-1} \sum_{\substack{p-1 \\
p-1}}^{p-1} 1 \\
& =1+4(p-2)(p-1)+\sum_{\substack{a=2 \\
(e-1)(e b-a) \equiv 0(\bmod p)}}^{p-1} \sum_{b=2}^{p-1} \sum_{e=1}^{p-1} 1 \\
& =1+4(p-2)(p-1)+2(p-2)^{2}-(p-2)=1+3(p-2)(2 p-3) \text {. }
\end{aligned}
$$

Third case: if $a+b+c \equiv d+e+1 \equiv 0(\bmod p)$ and $a^{2}+b^{2}+c^{2} \equiv d^{2}+e^{2}+1(\bmod p)$, then we have

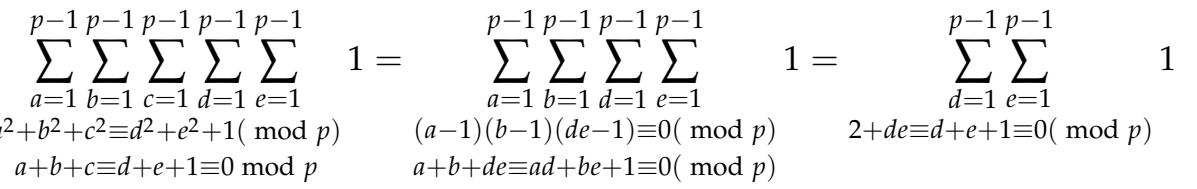

$$
\begin{aligned}
& a b c \equiv d e(\bmod p) \\
& +2 \sum_{\substack{b=2 \\
2+b \equiv d+b \bar{d}+1 \equiv 0(\bmod p)}}^{p-1} \sum_{d=1}^{p-1} 1+2 \sum_{\substack{b=2 \\
1+b+d e \equiv d+b e+1 \equiv 0(\bmod p)}}^{p-1} \sum_{\substack{d=1 \\
c-1}}^{p-1} 1+\sum_{\substack{a=2 \\
a+b+1 \equiv a d+b \bar{d}+1 \equiv 0(\bmod p)}}^{p-1} \sum_{b=2}^{p-1} \sum_{d=1}^{p-1} 1 \\
& (d e-1, p)=1 \\
& =2+4+2(p-4+p-5)+p-4+p-5=3(2 p-7) \text {. }
\end{aligned}
$$


Summarizing (3)-(5) we have the identity

$$
\begin{aligned}
& \sum_{a=1}^{p-1} \sum_{b=1}^{p-1} \sum_{c=1}^{p-1} \sum_{d=1}^{p-1} \sum_{e=1}^{p-1} 1=\sum_{a=1}^{p-1} \sum_{b=1}^{p-1} \sum_{c=1}^{p-1} \sum_{d=1}^{p-1} \sum_{e=1}^{p-1} 1 \\
& a^{3}+b^{3}+c^{3} \equiv d^{3}+e^{3}+1(\bmod p) \quad(a+b+c)\left(a^{2}+b^{2}+c^{2}-d^{2}-e^{2}-1\right) \equiv 0(\bmod p) \\
& a b c \equiv d e(\bmod p) \quad a b c \equiv d e(\bmod p) \\
& a+b+c \equiv d+e+1(\bmod p) \quad a+b+c \equiv d+e+1(\bmod p)
\end{aligned}
$$

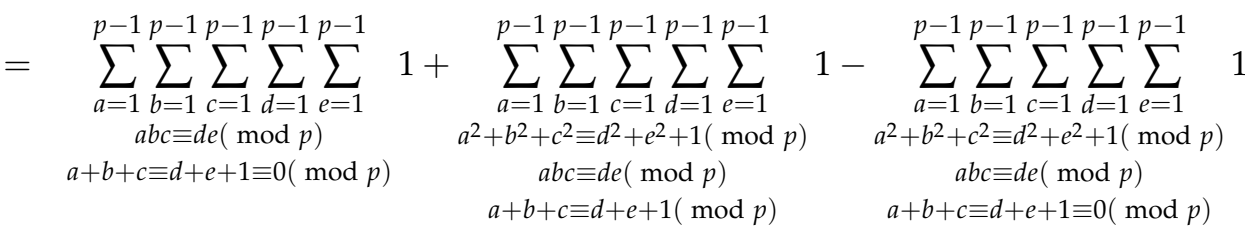

$$
\begin{aligned}
& =(p-2)^{2}+1+3(p-2)(2 p-3)-3(2 p-7)=7 p^{2}-31 p+44 \text {. }
\end{aligned}
$$

This proves Lemma 3.

Lemma 4. Let $p$ be an odd prime, then we have the identity

$$
\begin{aligned}
& \sum_{\substack{a=1 \\
a+b+c \equiv 1 \\
a-1}}^{p-1} \sum_{c=1}^{p-1} \sum_{d=1}^{p-1} \sum_{e=1}^{p-1} 1=6 p^{2}-21 p+19 . \\
& a b c \equiv d e(\bmod p) \\
& \bar{a}+\bar{b}+\bar{c} \equiv \bar{d}+\bar{e}+1(\bmod p)
\end{aligned}
$$

Proof. It is obvious that the conditions $a+b+c \equiv d+e+1(\bmod p), \bar{a}+\bar{b}+\bar{c} \equiv \bar{d}+\bar{e}+1(\bmod p)$ and $a b c \equiv d e(\bmod p)$ are equivalent to $a+b+c \equiv d+e+1 \bmod p, a b+a c+b c \equiv d e+d+e(\bmod p)$ and $a b c \equiv d e(\bmod p)$. These conditions are equivalent to $a+b+c \equiv d+e+1 \bmod p, a b+a c+b c+1 \equiv$ $a b c+a+b+c(\bmod p)$ and $a b c \equiv d e(\bmod p)$, or they are equivalent to $(a-1)(b-1)(c-1) \equiv$ $0(\bmod p)$ and $a+b+c \equiv d+e+1(\bmod p)$ and $a b c \equiv d e(\bmod p)$. From these we can easily obtain

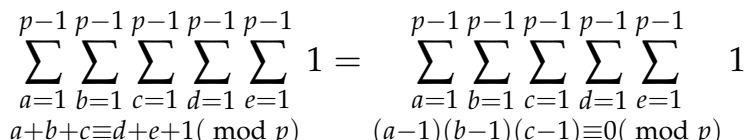

$$
\begin{aligned}
& a b c \equiv d e(\bmod p) \quad a b c \equiv d e(\bmod p) \\
& \bar{a}+\bar{b}+\bar{c} \equiv \bar{d}+\bar{e}+1(\bmod p) \quad a+b+c \equiv d+e+1(\bmod p)
\end{aligned}
$$

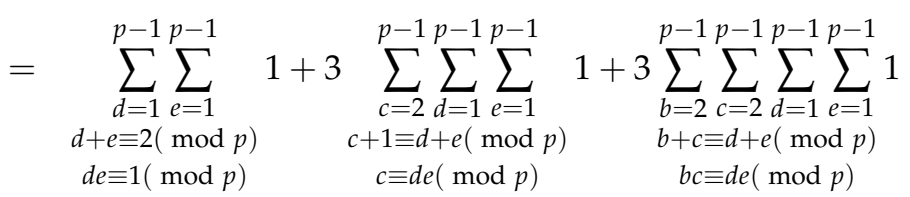

$$
\begin{aligned}
& =1+3 \sum_{c=2}^{p-1} \sum_{d=1}^{p-1} \sum_{e=1}^{p-1} 1+3 \sum_{b=2}^{p-1} \sum_{c=2}^{p-1} \sum_{d=1}^{p-1} \sum_{e=1}^{p-1} 1 \\
& d e+1 \equiv d+e(\bmod p) \quad b^{2}+d e \equiv(d+e) b(\bmod p) \\
& c \equiv \operatorname{de}(\bmod p) \quad b c \equiv d e(\bmod p)
\end{aligned}
$$

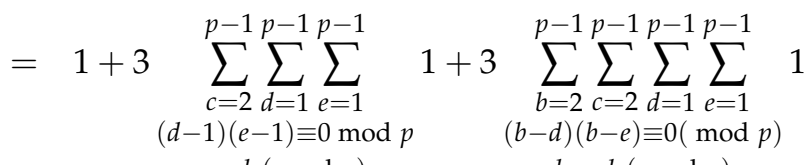

$$
\begin{aligned}
& c \equiv d e(\bmod p) \quad b c \equiv d e(\bmod p) \\
& =1+6(p-2)+3\left(2(p-2)^{2}-(p-2)\right)=6 p^{2}-21 p+19 \text {. }
\end{aligned}
$$

This finishes the proof of Lemma 4. 


\section{Proofs of the Theorems}

Proof of Theorem 1. It is not difficult to complete the proofs of our theorems. At first, we are going to prove Theorem 1. Suppose that $3 \nmid(p-1)$, then from Lemma 1, Lemma 3, the trigonometric identity

$$
\sum_{a=0}^{p-1} e\left(\frac{m a}{p}\right)= \begin{cases}p, & \text { if } p \mid m ; \\ 0, & \text { if } p \nmid m\end{cases}
$$

and the orthogonality of characters $\bmod p$, we obtain

$$
\begin{aligned}
& \sum_{\bmod } \sum_{p}^{p-1}\left|\sum_{m=1}^{p-1} \chi(a) e\left(\frac{m a^{3}+a}{p}\right)\right|^{6} \\
& =p(p-1) \sum_{\substack{a=1 \\
a^{3}+b^{3}+c^{3} \equiv d^{3}+e^{3}+f^{3}(\bmod p)}}^{p-1} \sum_{c=1}^{p-1} \sum_{\substack{p-1 \\
p-1}}^{p-1} e\left(\frac{a+b+c-d-e-f}{p}\right) \\
& a b c \equiv \operatorname{def}(\bmod p) \\
& =p(p-1) \sum_{\substack{a=1 \\
a^{3}+b^{3}+c^{3} \equiv d^{3}+e^{3}+1(\bmod p)}}^{p-1} \sum_{c=1}^{p-1} \sum_{d=1}^{p-1} \sum_{e=1}^{p-1} \sum_{f=1}^{p-1} e\left(\frac{f(a+b+c-d-e-1)}{p}\right) \\
& a b c \equiv d e(\bmod p)
\end{aligned}
$$

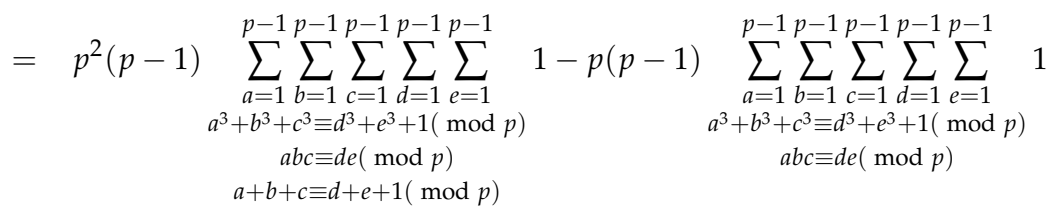

$$
\begin{aligned}
& =p^{2}(p-1)\left(7 p^{2}-31 p+44\right)-p(p-1)\left(p^{3}-3 p^{2}+5 p-5\right) \\
& =p(p-1)\left(6 p^{3}-28 p^{2}+39 p+5\right) \text {. }
\end{aligned}
$$

This finishes of the proof of Theorem 1.

Proof of Theorem 2. According to (6), Lemma 2 and Lemma 4, we obtain

$$
\begin{aligned}
& \sum_{\chi \bmod } \sum_{p}^{p-1}\left|\sum_{a=1}^{p-1} \chi(a) e\left(\frac{m a+\bar{a}}{p}\right)\right|^{6} \\
& =p(p-1) \sum_{a=1}^{p-1} \sum_{b=1}^{p-1} \sum_{c=1}^{p-1} \sum_{d=1}^{p-1} \sum_{e=1}^{p-1} \sum_{f=1}^{p-1} e\left(\frac{\bar{a}+\bar{b}+\bar{c}-\bar{d}-\bar{e}-\bar{f}}{p}\right) \\
& \begin{aligned}
a+b+c & \equiv d+e+f(\bmod p) \\
a b c & \equiv \operatorname{def}(\bmod p)
\end{aligned} \\
& \begin{array}{c}
p(p-1) \sum_{a=1}^{p-1} \sum_{b=1}^{p-1} \sum_{c=1}^{p-1} \sum_{d=1}^{p-1} \sum_{e=1}^{p-1} \sum_{f=1}^{p-1} e\left(\frac{f(\bar{a}+\bar{b}+\bar{c}-\bar{d}-\bar{e}-1)}{p}\right) \\
a+b+c \equiv d+e+1(\bmod p) \\
a b c \equiv d e(\bmod p)
\end{array} \\
& =p^{2}(p-1) \sum_{\substack{a=1 \\
a+b+c \equiv d}}^{p-1} \sum_{b=1}^{p-1} \sum_{c=1}^{p-1} \sum_{d=1}^{p-1} \sum_{e=1}^{p-1} 1-p(p-1) \sum_{\substack{a=1 \\
a+b+c \equiv d+e+1(\bmod p)}}^{p-1} \sum_{b=1}^{p-1} \sum_{c=1}^{p-1} \sum_{d=1}^{p-1} \sum_{e=1}^{p-1} 1 \\
& a b c \equiv d e(\bmod p) \quad a b c \equiv d e(\bmod p) \\
& \bar{a}+\bar{b}+\bar{c} \equiv \bar{d}+\bar{e}+1(\bmod p) \\
& =p^{2}(p-1)\left(6 p^{2}-21 p+19\right)-p(p-1)\left(p^{3}-3 p^{2}+5 p-5\right) \\
& =p(p-1)\left(5 p^{3}-18 p^{2}+14 p+5\right) \text {. }
\end{aligned}
$$

Proof of Corollary 1 . Note that if $m=0$, then from the properties of the classical 
Gauss sums, we deduce that

$$
\left|\sum_{a=1}^{p-1} \chi(a) e\left(\frac{0 \cdot a^{3}+a}{p}\right)\right|=\left|\sum_{a=1}^{p-1} \chi(a) e\left(\frac{a}{p}\right)\right|= \begin{cases}\sqrt{p}, & \text { if } \chi \neq \chi_{0} \\ 1, & \text { if } \chi=\chi_{0}\end{cases}
$$

Hence, from (7) we can easily obtain

$$
\sum_{\chi \bmod p}\left|\sum_{a=1}^{p-1} \chi(a) e\left(\frac{0 \cdot a^{3}+a}{p}\right)\right|^{6}=p^{3}(p-2)+1=(p-1)\left(p^{3}-p^{2}-p-1\right) .
$$

Combining (8) and Theorem 1, we obtain

$$
\begin{aligned}
& \sum_{\chi \bmod } \sum_{m=1}^{p-1}\left|\sum_{a=1}^{p-1} \chi(a) e\left(\frac{m a^{3}+a}{p}\right)\right|^{6} \\
= & p(p-1)\left(6 p^{3}-28 p^{2}+39 p+5\right)-(p-1)\left(p^{3}-p^{2}-p-1\right) \\
= & (p-1)\left(6 p^{4}-29 p^{3}+40 p^{2}+6 p+1\right) .
\end{aligned}
$$

Taking

$$
\left|\sum_{a=1}^{p-1} \chi_{1}(a) e\left(\frac{m_{1} a^{3}+a}{p}\right)\right|=\max _{\substack{\chi \bmod p \\ 1 \leq m \leq p-1}}\left|\sum_{a=1}^{p-1} \chi(a) e\left(\frac{m a^{3}+a}{p}\right)\right|
$$

and combining (9) and (10), we may instantly deduce that

$$
\begin{aligned}
& \left.(p-1)^{2} \cdot|| \sum_{a=1}^{p-1} \chi_{1}(a) e\left(\frac{m_{1} a^{3}+a}{p}\right)\right|^{6} \geq \sum_{\chi \bmod } \sum_{m=1}^{p-1}\left|\sum_{a=1}^{p-1} \chi(a) e\left(\frac{m a^{3}+a}{p}\right)\right|^{6} \\
= & (p-1)\left(6 p^{4}-29 p^{3}+40 p^{2}+6 p+1\right) .
\end{aligned}
$$

It is clear that this inequality implies

$$
\left|\sum_{a=1}^{p-1} \chi_{1}(a) e\left(\frac{m_{1} a^{3}+a}{p}\right)\right| \geq\left(\frac{6 p^{4}-29 p^{3}+40 p^{2}+6 p+1}{p-1}\right)^{\frac{1}{6}}>6^{\frac{1}{6}} \cdot p^{\frac{1}{2}}-\frac{1}{\sqrt{p}} .
$$

This finishes of the proof of Corollary 1.

Proof of Corollary 2. We can also deduce Corollary 2 easily. In other words, we have the inequality

$$
\max _{\substack{\chi \bmod p \\ 1 \leq m \leq p-1}}\left|\sum_{a=1}^{p-1} \chi(a) e\left(\frac{m a+\bar{a}}{p}\right)\right|>5^{\frac{1}{6}} \cdot p^{\frac{1}{2}}-\frac{1}{\sqrt{p}} .
$$

The proofs of our results have been completed.

\section{Conclusions}

The main results of this paper are two theorems and two corollaries. The theorems give two exact computational formulae for the sixth power mean of the generalized Gauss sums and generalized Kloosterman sums. The corollaries give two sharper lower bound estimates for the generalized Gauss sums and generalized Kloosterman sums. In addition, as some notes of our results, we also proposed two thoughtful open problems. These results profoundly reveal the law of the value distribution of the 
generalized Gauss sums and generalized Kloosterman sums, which can also be used for references in the study of similar problems.

Author Contributions: All authors have equally contributed to this work. All authors read and approved the final manuscript.

Funding: This work is supported by the N. S. F. (11771351) and (11826205) of China.

Acknowledgments: The authors would like to thank the editors and referees for their very helpful and detailed comments, which have significantly improved the presentation of this paper.

Conflicts of Interest: The authors declare that there are no conflicts of interest regarding the publication of this paper.

\section{References}

1. Iwaniec, H. Topics in Classical Automorphic Forms. Grad. Stud. Math. 1997, 17, 61-63.

2. Zhang, W.P. On the fourth and sixth power mean of the classical Kloosterman sums. J. Number Theory 2011, 131, 228-238.

3. Zhang, W.P. On the fourth power mean of the general Kloosterman sums. Indian J. Pure Appl. Math. 2004, 35, 237-242. [CrossRef]

4. Zhang, W.P. On the fourth power mean of the general Kloosterman sums. J. Number Theory 2016, 169, 315-326. [CrossRef]

5. Zhang, W.P.; Shen, S.M. A note on the fourth power mean of the generalized Kloosterman sums. J. Number Theory 2017, 174, 419-426. [CrossRef]

6. Zhang, H.; Zhang, W.P. The fourth power mean of two-term exponential sums and its application. Math. Rep. 2017, 19, 75-81.

7. Zhang, W.P.; Han, D. On the sixth power mean of the two-term exponential sums. J. Number Theory 2014, 136, 403-413. [CrossRef]

8. Duan, R.; Zhang, W.P. On the fourth power mean of the generalized two-term exponential sums. Math. Rep. Unpublished work.

9. Weil, A. On some exponential sums. Proc. Natl. Acad. Sci. USA 1948, 34, 203-210. [CrossRef] [PubMed]

10. Han, D. A Hybrid mean value involving two-term exponential sums and polynomial character sums. Czechoslov. Math. J. 2014, 64, 53-62.

11. Chern, S. On the power mean of a sum analogous to the Kloosterman sum. Bull. Math. Soc. Sci. Math. Roum. 2019, 62, 77-92.

12. Zhang, T.P. On mixed two-term exponential sums. J. Korean Math. Soc. 2010, 47, 1107-1122.

13. Zhang, W.P.; Li, X.X. The fourth power mean of the general 2-dimensional Kloostermann sums mod $p$. Acta Math. Sinica Engl. Ser. 2017, 33, 861-867. [CrossRef]

14. Du, X.Y. The hybrid power mean of two-term exponential sums and character sums. Acta Math. Sinica (Chin. Ser.) 2016, 59, 309-316.

15. Lv, X.X.; Zhang, W.P. A new hybrid power mean involving the generalized quadratic Gauss sums and sums analogous to Kloosterman sums. Lith. Math. J. 2017, 57, 359-366. [CrossRef]

16. Wang, X.Y.; Li, X.X. One kind sixth power mean of the three-term exponential sums. Open Math. 2017, 15, 705-710. [CrossRef]

17. Zhang, Y.T. Bounded gaps between primes. Ann. Math. 2014, 179, 1121-1174. [CrossRef]

18. Apostol, T.M. Introduction to Analytic Number Theory; Springer: New York, NY, USA, 1976.

(c) 2019 by the authors. Licensee MDPI, Basel, Switzerland. This article is an open access article distributed under the terms and conditions of the Creative Commons Attribution (CC BY) license (http:// creativecommons.org/licenses/by/4.0/). 